\title{
Cyclotron amplification of whistler-mode waves: A parametric study relevant to discrete VLF emissions in the Earth's magnetosphere
}

\author{
D. L. Pasmanik, ${ }^{1}$ A. G. Demekhov, ${ }^{1}$ D. Nunn, ${ }^{2}$ V. Y. Trakhtengerts, ${ }^{1}$ and M. J. Rycroft ${ }^{3}$ \\ Received 8 August 2001; revised 10 December 2001; accepted 11 December 2001; published 3 August 2002.
}

[1] We study the amplification of ducted whistler-mode waves in the Earth's magnetosphere by the cyclotron instability for different types of energetic electron distributions in velocity space. Particular attention is paid to the comparison between cases of smooth distribution functions and those with sharp gradients ("steps") in velocity space, which arise naturally owing to interactions with noise-like VLF emissions. We show that step-like features greatly favor the amplification of whistler-mode waves propagating along the Earth's magnetic field line and hence the generation of narrowband VLF emissions even if the electron anisotropy is only moderate. The results obtained are discussed in light of observations of discrete VLF emissions from the magnetosphere, in particular those of Bell et al. [2000]. INDEX TERMS: 2720 Magnetospheric Physics: Energetic particles, trapped; 2730 Magnetospheric Physics: Magnetosphere - inner; 0654 Electromagnetics: Plasmas; 2772 Magnetospheric Physics: Plasma waves and instabilities; KEYWORDS: whistlers, amplification, anisotropic, electrons, step-like, chorus

\section{Introduction}

[2] Satellite experiments on VLF chorus and triggered (narrowband) emissions demonstrate the occurrence of very large amplification of the primary whistler wave during the process of cyclotron interaction with energetic electrons in the Earth's magnetosphere. This amplification reaches 40 to 60 dB [Carlson et al., 1990; Omura et al., 1991; Bell et al., 2000]. Theoretical studies [Nunn and Smith, 1996; Smith and Nunn, 1998], also show that for effective nonlinear particle trapping and following generation of a triggered signal, rather high amplitude of the primary whistler signal near the equatorial region is required. Such values of the wave amplitude can be reached due to a cyclotron interaction with trapped particles as the initial wave propagates along the magnetic field line.

[3] It is very difficult to explain such a large amplification using currently available satellite data on energetic electrons in the Earth's magnetosphere. Estimates show that the observed trapped electron flux is insufficient to obtain these amplification values if one assumes a typical smooth distribution with moderate values of transverse anisotropy, i.e. the with the perpendicular temperature not too much exceeding the parallel temperature. Note that the quasi-stable electron population in the inner magnetosphere is unlikely to have the beam-like distribution with narrow spread. On the other

\footnotetext{
${ }^{1}$ Institute of Applied Physics, Russian Academy of Sciences, Nizhny Novgorod, Russia.

${ }^{2}$ Department of Electronics and Computer Science, Southampton University, Southampton, UK.

${ }^{3}$ Faculty of Computer Sciences and Engineering, De Montfort University, Leicester, UK.
}

Copyright 2002 by the American Geophysical Union. 0148-0227/02/2001JA000256\$09.00 hand, assumption that the distribution function has a sharp gradient (step-like feature) in velocity space allows one to explain recent observations of energetic electrons and whistler-mode waves [Trakhtengerts et al., 2001].

[4] In this paper, we consider a step in the distribution function of energetic electrons over the field-aligned velocity component $v_{\|}$. This step appears in a natural way as the cyclotron instability develops, and serves as the boundary between resonant and non-resonant electrons interacting with a whistler-mode hiss (broadband) signal [Bespalov and Trakhtengerts, 1986; Tagirov et al., 1986; Trakhtengerts et al., 1996; Trakhtengerts and Rycroft, 2000].

[5] In contrast to the case of smooth energetic electron distributions, which was studied in a huge number of papers, the whistler cyclotron instability with a step-like electron distribution has been considered by not many authors [Nunn and Sazhin, 1991; Villalón and Burke, 1997; Hobara et al., 1998]. This is probably explained by the absence of obvious experimental evidence for this type of distributions. Indeed, to detect such feature, the instruments with very fine energy, pitch-angle, and temporal resolution are required. Moreover, measurements should be made in the region close to the equatorial plane, where the step is formed. Nevertheless, some indications of a step in the distribution function were revealed in OGO-5 satellite data [Lyons et al., 1972]. Unfortunately, the energy and pitch angle resolution of these data ( $\sim 10 \%$ and $\sim 5$ to $10^{\circ}$, respectively) were not enough to reconstruct the exact width and dynamic features of the step.

[6] The formation of this step-like feature in the energetic electron distribution was explained by Trakhtengerts et al. [1996]. Nunn and Sazhin [1991] and Villalón and Burke [1997] analysed the wave growth rate for this type of distribution. It is important to note that, in the case when waves propagate along the inhomogeneous geomagnetic field, use of the local value of the growth rate for the 
analysis of wave generation is not quite correct. Rather, the one-hop amplification of a wave packet determined by the growth of wave amplitude along the entire path of the wave packet between magnetically conjugate regions of the ionosphere is required. A rigorous analysis of this problem was performed by Hobara et al. [1998].

[7] The main goal of this paper is to extend previous studies by making a detailed comparison of the amplification rates for both smooth and step-like distribution functions, for similar values of parameters such as energetic electron number density, energy, and transverse (pitch angle) anisotropy. In particular, we show that choosing the parameters according to recent observations of triggered VLF emissions [Bell et al., 2000] yields a sufficient amplification rate to explain these triggered emissions for the step-like distribution function, whereas that is impossible if a smooth distribution with the same parameters is assumed.

\section{Cyclotron Amplification of Whistler-Mode Waves}

[8] The one-hop amplification of a wave packet, i.e., the ratio of wave amplitudes after and before it propagates through the interaction region which is considered to be near the equatorial plane of the magnetosphere, is defined as

$$
\Gamma(\omega, \vec{k})=\ln \left[\frac{A(l)}{A(-l)}\right]=\int_{-l}^{l} \frac{\gamma(\omega, \vec{k}, z)}{V_{g \|}} d z
$$

where $\omega$ and $\vec{k}$ are the frequency and wave vector of the whistler-mode wave, $z$ is the coordinate along the magnetic field line (with the origin being at the equatorial plane), $\pm l$ are coordinates of the feet of the field line in the conjugate regions of the ionosphere, $\gamma(\omega, \vec{k}, z)$ is the local growth rate, $V_{g \|}$ is the component of the group velocity parallel to the geomagnetic field $\vec{H}$, and $A$ is the wave amplitude.

[9] To simplify the analysis, we shall restrict our consideration to the case of ducted whistler wave propagation, when $\vec{k} \| \vec{H}$. The case of oblique wave propagation demands more complex analysis, and will be considered in another paper. In our case, the amplification can be written as [Trakhtengerts et al., 1996]

$$
\begin{aligned}
\Gamma= & \frac{\pi^{2} e^{2}}{c m^{2}} \int_{W_{R L}}^{\infty} \int_{0}^{W-W_{R L}} \\
& \cdot\left[\frac{I_{\perp} H^{2} d W d I_{\perp}}{N_{w}\left(W-I_{\perp} H\right)}\left(\frac{\partial F_{0}}{\partial W}+\frac{e}{m c \omega} \frac{\partial F_{0}}{\partial I_{\perp}}\right) \mathcal{I}\right]_{z_{\mathrm{st}}\left(W, I_{\perp}, \omega\right)}
\end{aligned}
$$

where the energetic electrons' energy $W=m\left(v_{\perp}^{2}+v_{\|}^{2}\right) / 2$ and the first adiabatic invariant $I_{\perp}=m v_{\perp}^{2} / 2 H$ are used as velocity-space variables, $v_{\perp}$ and $v_{\|}$are, respectively, the components of electron velocity parallel and perpendicular to the geomagnetic field $\vec{H}, F_{0}$ is the distribution function of the energetic electrons, and $N_{w}=k c / \omega$ is the whistler-mode wave refractive index. The factor $\mathcal{I}$ is defined by

$$
\mathcal{I}=\left|\int_{-l}^{l} \exp \left(i \int_{0}^{z} \frac{\Delta}{v_{\|}} d z^{\prime \prime}\right) d z\right|^{2}
$$

where $\Delta=\omega-\omega_{H}-k v_{\|}$is the cyclotron phase mismatch, and $\omega_{H}$ is the local electron gyrofrequency. The integrals in (2) are taken at the stationary point $z_{\text {st }}$, which is found from the equality $\Delta=0$, the exact Doppler shifted cyclotron resonance condition. The integration limits are determined from the condition that the lowest resonance energy $W_{R L}=$ $m v_{R L}^{2} / 2$ is achieved at the equator $\left(v_{R L}=\left(\omega_{H L}-\omega\right) / k_{L}\right.$; hereafter the subscript $L$ refers to the values in the equatorial plane). The electron charge and mass are, respectively, $e$ and $m ; c$ is the velocity of light in free space.

[10] We shall consider two different types of distribution function for the energetic electrons. The first is a smooth anisotropic distribution, which we choose in the form [Cornilleau-Wehrlin et al., 1985]

$$
F_{\text {smooth }}=B_{\text {smooth }} \frac{n_{h}}{v_{0}^{3}}\left(\sin ^{2} \Theta_{L}\right)^{\alpha}\left(\frac{W_{0}}{W+W_{1}}\right)^{\beta} .
$$

Here $n_{h}$ is the density of energetic electrons in the equatorial plane, $\Theta_{L}$ is electron pitch angle in the equatorial plane, $\alpha$ is the anisotropy index (the ratio of the perpendicular and the parallel temperatures of the energetic electrons). The power-law energy spectrum is determined by characteristic electron energy $W_{0}=m v_{0}^{2} / 2$ and parameters $\beta$ and $W_{1}$, which quantify the ratio between the number of electrons with high and low energies: as $W_{1}$ increases or $\beta$ decreases the number of the high energy particles grows. The parameter $W_{1}$ ensures that the distribution is finite at $W \rightarrow 0$. The normalization constant $B_{\text {smooth }}$ is given by

$$
B_{\text {smooth }}^{-1}=\frac{1}{4} \int_{0}^{\infty} x^{-\alpha}\left(x+x_{1}\right)^{-\beta} \int_{0}^{x} \frac{y^{\alpha}}{\sqrt{x-y}} d y d x
$$

where $y=I_{\perp} H_{L} / W_{0}, x=W / W_{0}, x_{1}=W_{1} / W_{0}$.

[11] The second type of distribution is a distribution with a step-like feature, which appears as the cyclotron instability of a smooth energetic electron distribution with a whistler hiss band develops; the position of the step corresponds to the boundary between resonant and nonresonant electrons [Trakhtengerts et al., 1996]. For this type of distribution

$$
F_{\text {step }}(I, W)=B_{\text {step }} n_{h} \cdot \chi\left(W_{\star}-W_{\|}\right) I_{\perp} e^{-W / W_{0}}
$$

where $\chi(x)$ is the Heaviside unit function

$$
\chi(x)= \begin{cases}1, & x>0 \\ 0, & x \leq 0\end{cases}
$$

$W_{\|}=W-I_{\perp} H, W_{\star}=m v_{\star}^{2} / 2$, and $v_{\star}$ corresponds to the velocity at the boundary between resonant and nonresonant electrons. The normalization constant $B_{\text {step }}=\left(\frac{m}{2 \pi W_{0}}\right)^{3 / 2}$ $H_{L} / W_{0}$ The exact form of the smooth part of the distribution used in (6) is not important, because its contribution to the amplification is much smaller than that of the "step" part.

[12] Substituting (4) into (2), we find the following expression for whistler wave amplification by a smooth energetic electron distribution: 


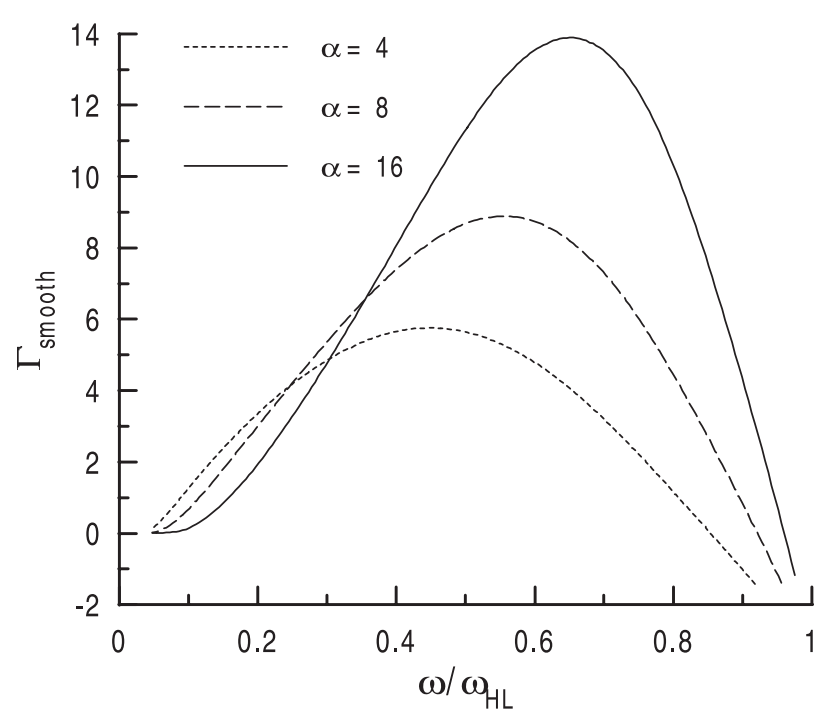

(a)

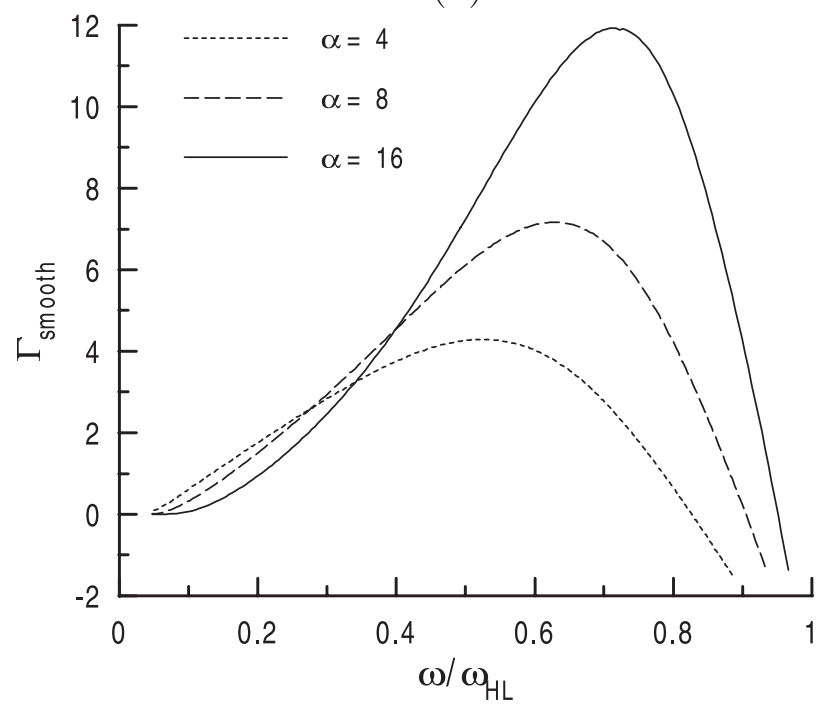

(b)

Figure 1. One-hop amplification $\Gamma_{\text {smooth }}$ of a whistlermode wave by energetic electrons with a smooth anisotropic distribution function of the energetic electrons (8) as a function of wave frequency normalized to the equatorial gyrofrequency, for different values of the anisotropy index $\alpha$ and energy parameter $W_{1}$ : (a) $W_{1} / W_{0}=0.1$; (b) $W_{1} / W_{0}=$ 0.5 . The values of the other parameters are $\beta=-2.5, v_{0}=$ $8 \cdot 10^{4} \mathrm{~km} / \mathrm{s}, n_{h}=10^{-2} \mathrm{~cm}^{-3}, L=3.4, \omega_{p L}=9 \cdot 10^{5} \mathrm{~Hz}$.

$$
\begin{aligned}
\Gamma_{\text {smooth }}= & \frac{\pi B_{\text {smooth }}}{4 N_{w}} \frac{n_{h}}{n_{c}} \frac{\omega_{p}^{2} v_{0}}{c} \int_{x_{\min }}^{\infty} d x \int_{0}^{x-x_{\min }} y d y \frac{H_{\mathrm{st}}^{2}}{H_{L}^{2}} \frac{\mathcal{I}(y / x)^{\alpha}}{v_{\| s t}^{2}\left(x+x_{1}\right)^{\beta}} \\
& \cdot\left(-\frac{\alpha}{x}-\frac{\beta}{x+x_{1}}+\frac{\omega_{H L}}{\omega} \frac{\alpha}{y}\right)
\end{aligned}
$$

where $x_{\min }=W_{R L} / W_{0}, v_{\| s t}=v_{0} \sqrt{x-y H_{\mathrm{st}} / H L}, H_{\mathrm{st}}=H\left(z_{\mathrm{st}}\right)$, the subscript st refers to the stationary point introduced in (2), $n_{c}$ is the cold plasma density, and $\omega_{p}$ is the electron plasma frequency. To calculate $\mathcal{I}$ we use the stationary phase method [Trakhtengerts et al., 1996]:

$$
\mathcal{I}=\left|\frac{\pi v_{\|}}{\partial \Delta / \partial z}\right|_{z_{\mathrm{st}}}
$$

where

$$
\left(\frac{\partial \Delta}{\partial z}\right)_{z_{\mathrm{st}}}=\frac{z_{\mathrm{st}}}{a^{2}}\left(2 \omega_{H L}+\frac{\omega H_{L}}{H_{\mathrm{st}}}+\frac{k v_{\perp_{L}}^{2} H_{L}}{\left|v_{\| s t}\right|}\right) .
$$

Here we have used the following approximation of the whistler wave refractive index in case of propagation parallel to magnetic field line

$$
N_{w}=\omega_{p} / \sqrt{\omega\left(\omega_{H}-\omega\right)}
$$

and the parabolic approximation for both the magnetic field strength and cold plasma density distribution along the geomagnetic field line close to the geomagnetic equator:

$$
\frac{H}{H_{L}}=\frac{n_{c}}{n_{c_{L}}}=\left(1+\frac{z^{2}}{a^{2}}\right)
$$

where $a=\sqrt{2} R_{0} L / 3$ is the characteristic scale of the Earth's magnetic field at the magnetic shell $L$, and $R_{0}$ is the Earth's radius.

[13] Using (11) and (12), we have, from $\Delta=0$, the following cubic equation for the value of the gyrofrequency at the stationary point $\omega_{H_{s t}}$ :

$$
\omega_{H_{\mathrm{st}}}^{3}+\omega_{H_{\mathrm{st}}}^{2} \omega\left(\frac{\omega_{p L}^{2} v_{0}^{2}}{\omega_{H L}^{2} c^{2}} y-3\right)+\omega_{H_{\mathrm{st}}} \omega\left(3 \omega-\frac{\omega_{p L}^{2} v_{0}^{2}}{\omega_{H L} c^{2}} x\right)-\omega^{3}=0 .
$$

[14] In the case of a step-like distribution (6), we have, from (2),

$\begin{array}{ll}\Gamma_{\text {step }}=\frac{n_{h}}{\sqrt{\pi} n_{c} N_{w}} \frac{\omega_{p}^{2} v_{0}}{c} \frac{\left(\omega_{H}-\omega\right)}{\omega} e^{-W_{\star} / W_{0}} \int_{0}^{\infty} \frac{H_{\text {st }}^{2}}{H_{L}^{2}} \frac{y^{2} d y}{v_{\| s t}^{2}} e^{-y} \mathcal{I}, & \text { if } v_{\star} \geq v_{R L} \\ \Gamma_{\text {step }}=0 & \text {,if } v_{\star}<v_{R L}\end{array}$

where now $v_{\| s t}=\sqrt{v_{\star}^{2}+y v_{0}^{2}\left(1-H_{\text {st }} / H L\right)}$, and the value for $\omega_{H_{\mathrm{st}}}$ is the solution of $(13)$ for $x=y+v_{\star}^{2} / v_{0}^{2}$.

[15] The expression (9) for $\mathcal{I}$ is valid only for a smooth distribution of energetic electrons, when the dispersion in $v_{\|}$ is large enough [Hobara et al., 1998]:

$$
\Delta v_{\|} / v_{\|} \gg(k a)^{-2 / 3}
$$

This condition means that rather small part of particles is at cyclotron resonance with a wave at the stationary point $z_{\text {st }}$. For parameters used in our calculations (see bellow) $(k a)^{-2 / 3}$ $\sim 10^{-2}$.

[16] Thus, in the case of a step-like distribution function, we should use more accurate expression for $\mathcal{I}$. For that a 


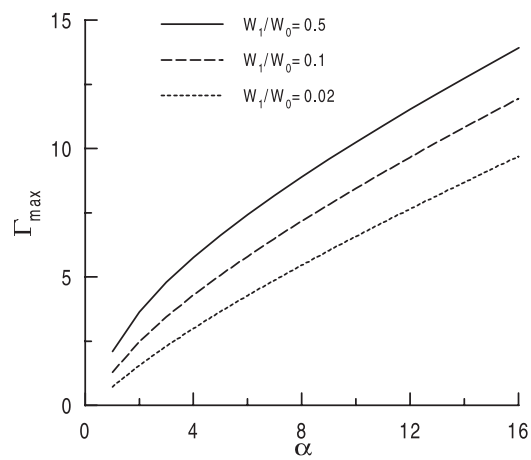

(a)

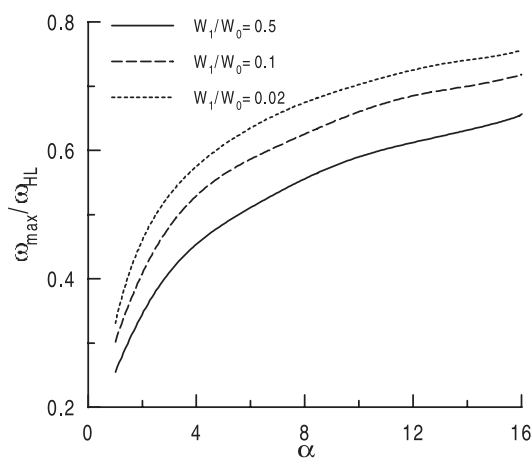

(b)

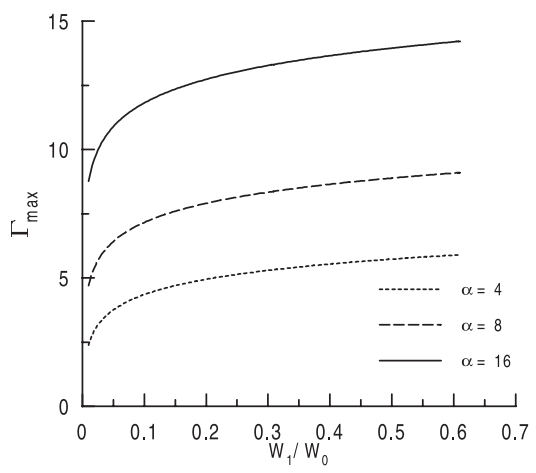

(c)

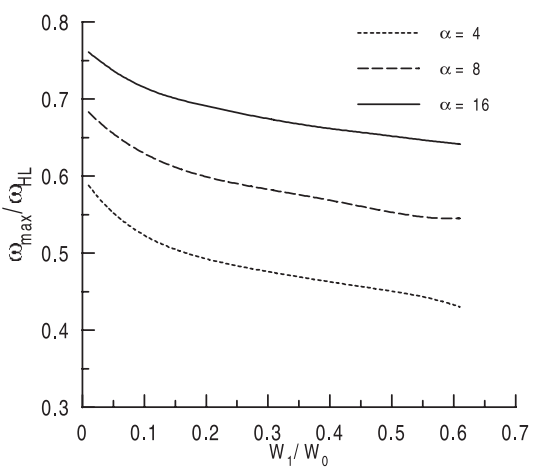

(d)

Figure 2. The maximum one-hop amplification $\Gamma_{\max }$ (panels a,c) and the corresponding frequency $\omega_{\max }$ ( panels b,d) of a whistler-mode wave by energetic electrons with a smooth anisotropic distribution (8). The different curves correspond to different values of the anisotropy index $\alpha$ (panels a,b) and the parameter $W_{1}$ (panels c,d); all the other parameters are as shown in Figure 1.

second order expansion of the spatial dependence of $\Delta$ near the stationary point $z_{\text {st }}$ in the calculation of integral (3) must be used. Following Hobara et al. [1998] we have:

$$
\mathcal{I}=4 \pi^{2} a^{2} b^{-2 / 3} \mathrm{Ai}^{2}(-P(y))
$$

where $P(y)=b^{2 / 3}\left(z_{\mathrm{st}} / a\right)^{2}$, Ai is Airy function, and

$$
b=\frac{\omega_{H L} a}{v_{\| s t}}\left(1+\frac{\omega}{2 \omega_{H_{\mathrm{st}}}}+\frac{k v_{0}^{2}}{2 v_{\| L} \omega_{H L}} y\right) .
$$

\section{Results}

[17] The results of numerical calculations for the expressions for one-hop whistler wave amplification, (8) and (14), are presented in Figures 1-4. In our calculations we have used the parameters corresponding to the experimental data presented by Bell et al. [2000]. These are: $L=3.4\left(a \approx 10^{4}\right.$ $\left.\mathrm{km}, \omega_{B L} / 2 \pi \approx 22 \mathrm{kHz}\right), n_{h}=10^{-2} \mathrm{~cm}^{-3}, \omega_{p_{L}}=9 \cdot 10^{5} \mathrm{~Hz}$, and $v_{0}=8 \cdot 10^{4} \mathrm{~km} / \mathrm{s}\left(W_{0} \approx 18 \mathrm{keV}\right)$.

[18] The dependence of the one-hop amplification $\Gamma_{\text {smooth }}$ on wave frequency $\omega$, for different values of the anisotropy index $\alpha$ and energy parameter $W_{1}$, is shown in Figures 1a and $1 \mathrm{~b}$. It is important to note, that in the case of a step-like distribution function, the profile of the energetic electron number density along the magnetic field line is similar to the case of a highly anisotropic smooth distribution (see
Figure 2 of Trakhtengerts et al. [2001]). Thus, to compare the smooth and step-like distributions with similar parameters, we use rather high values of the anisotropy index $\alpha$ in our calculations.

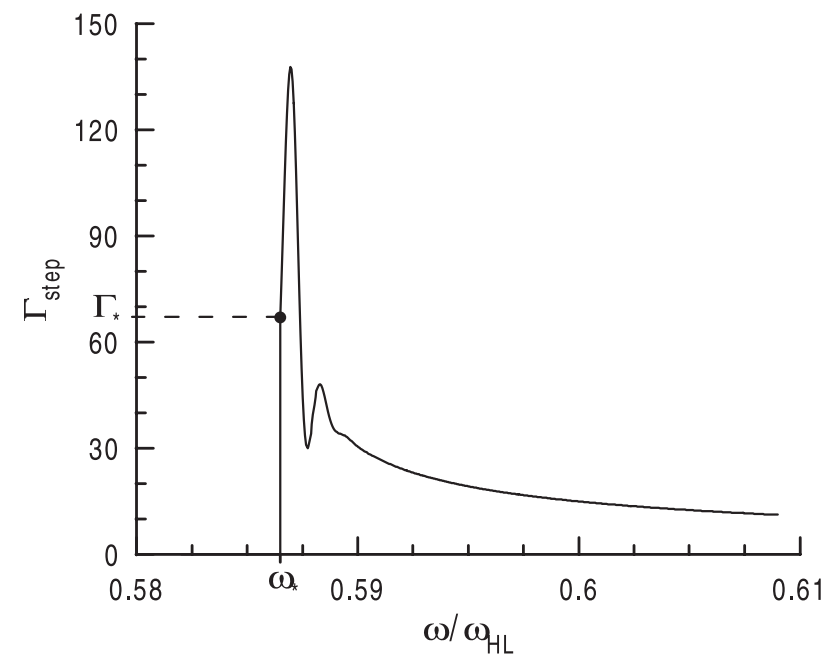

Figure 3. One-hop amplification $\Gamma_{\text {step }}$ of a whistler-mode wave by energetic electrons with a step-like distribution function for the energetic electrons (14); $v_{0}=8 \cdot 10^{4} \mathrm{~km} / \mathrm{s}$, $v_{\star} / v_{0}=0.2, n_{h}=10^{-2} \mathrm{~cm}^{-3}, L=3.4, \omega_{p L}=9 \cdot 10^{5} \mathrm{~Hz}$. The frequency $\omega_{\star}$ and corresponding value of $\Gamma_{\star}(19)$ are marked by the dot. 


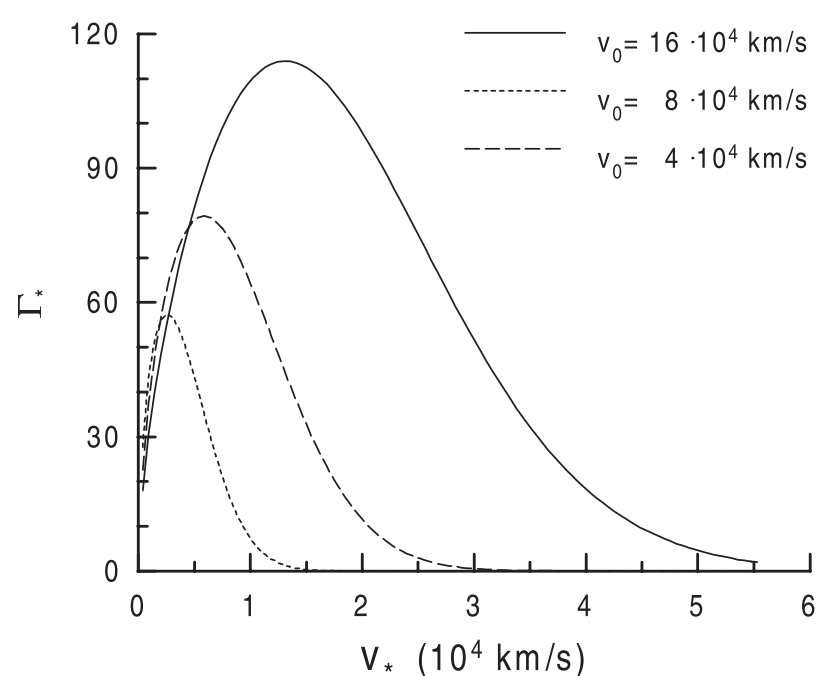

(a)

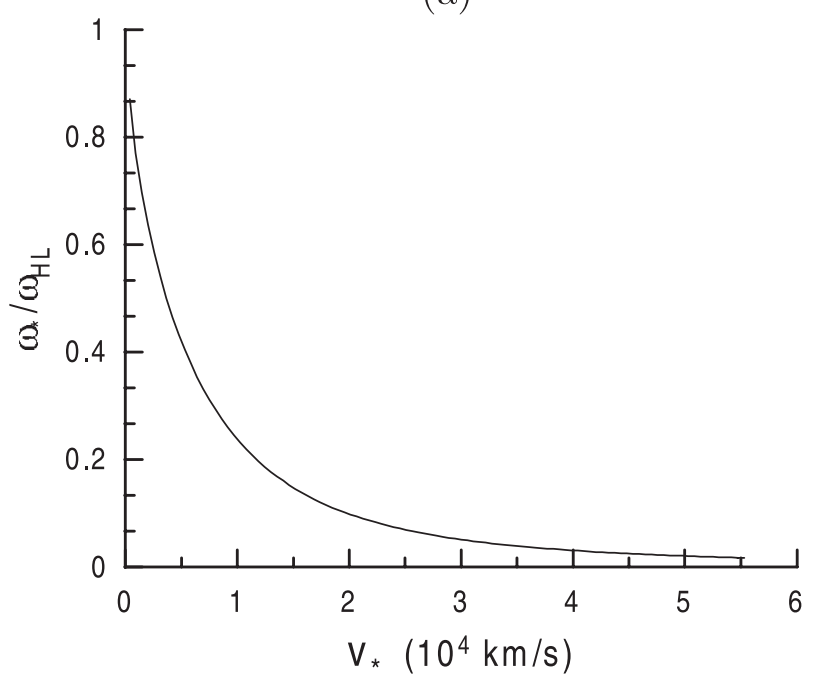

(b)

Figure 4. (a) Shown as a function of $v_{\star}$ is the one-hop amplification $\Gamma_{\star}$ of a whistler-mode wave by a step-like distribution of energetic electrons for the case when the step is in resonance with the wave at the equatorial plane. (b) Values of the wave frequency $\omega_{\star}$ corresponding to resonance with the step at the equatorial plane; other parameters have the values $n_{h}=10^{-2} \mathrm{~cm}^{-3}, L=3.4, \omega_{p L}=$ $9 \cdot 10^{5} \mathrm{~Hz}$, as before.

[19] It is seen that for a smooth distribution function the amplification occurs over a rather wide frequency band, from, say, 0.25 to $0.8 \omega_{H L}$. For a chosen distribution (4), the maximum value of the one-hop amplification increases as the anisotropy index $\alpha$ increases. As is mentioned above, the larger value of $W_{1}$ parameter corresponds to the distribution with more high-energy electrons. Thus, the number of resonant particles and, hence, the one-hop amplification increases with increase in $W_{1}$. For rather large values of this parameter $\left(W_{1} / W_{0}>1\right)$ the dependence of the amplification on $W_{1}$ becomes very weak. A decrease in $\beta$ leads to a similar variation in the distribution and amplification as an increase in $W_{1}$. So, the results for one particular value of this parameter are shown. The dependencies of the maximum amplification $\Gamma_{\max }$ and the corresponding frequency $\omega_{\max }$ on the anisotropy index $\alpha$ and parameter $W_{1}$ are presented in Figure 2. The one-hop amplification typically lies between 3 and 13 for the plasma parameters shown.

[20] The amplification in the case of a step-like distribution is shown in Figure 3. This plot demonstrates the typical dependence of the one-hop amplification on frequency, which exhibits one strong narrowband peak and nearby much weaker peaks. Varying the parameter values leads to change of the maximum amplification value and corresponding frequency, as illustrated in Figure 4.

[21] In contrast to the previous case (Figures 1 and 2), the one-hop amplification $\Gamma_{\text {step }}$ has a sharp maximum with a large value ( $\gtrsim 100)$; only a very narrow part of the whistler band is amplified $\left(\Delta \omega / \omega_{H L} \sim 0.01\right)$. The position of the maximum of the one-hop amplification is near the frequency $\omega_{\star}$ defined by the condition that the wave is in cyclotron resonance with the step exactly at the equatorial plane:

$$
v_{\star}=\frac{\omega_{H L}-\omega_{\star}}{k_{L}} .
$$

The oscillatory behavior of the amplification is due to the fact that the efficiency of cyclotron interaction, which is determined by the factor $\mathcal{I}$, depends on the wave-particle phase mismatch between two cyclotron resonance points $\pm z_{\text {st }}$ symmetrically located on either side of the equator. This mismatch is a function of the electron energy and pith-angle and wave frequency, so the $\mathcal{I}$ has the oscillatory dependence on $P(y)$, which results in oscillatory dependence of amplification on the frequency. This point is discussed in more detail by Hobara et al. [1998].

[22] From (17) we have

$$
\left(\frac{v_{\star} \omega_{p L}}{c \omega_{H L}}\right)^{2}=\frac{\omega_{H L}}{\omega_{\star}}\left(1-\frac{\omega_{\star}}{\omega_{H L}}\right)^{3}
$$

and the following expression for the one-hop amplification at the frequency $\omega_{\star}$ :

$$
\Gamma_{\star}=4 \pi^{3 / 2} A i^{2}(0) \frac{n_{h} \omega_{p L}^{2} a^{2} v_{0}}{n_{c} v_{\star} c^{2}} e^{-W_{\star} / W_{0}} \int_{0}^{\infty} b^{-2 / 3} y^{2} e^{-y} d y .
$$

The results of numerical computations of (19) and (18) are presented in Figure 4 for three values of $v_{0}=4,8$ or $16 \times$ $10^{4} \mathrm{~km} / \mathrm{s}$, i.e. for electron energies $W_{0} \approx 4.5,18,70 \mathrm{keV}$. As the value of the step velocity $v_{\star}$ grows, efficiency of cyclotron wave-particle interactions increases $\left(b^{-2 / 3}\right.$ term in (19)), but the number of resonant particles decreases $\left(e^{-W_{\star} / W_{0}}\right.$ term in (19)). Therefore the dependence of $\Gamma_{\star}$ on $v_{\star}$ has a maximum (Figure 4a). It should be mentioned that, according to our numerical analysis, the maximum of the one-hop amplification $\Gamma_{\text {step }}(14)$ is not exactly at the frequency $\omega_{\star}$ (see Figure 3, where the frequency $\omega_{\star}$ and corresponding value of $\Gamma_{\star}(19)$ are marked by the dot). The maximum is at a slightly higher frequency, by $\Delta \omega_{\star}<0.005$ $\omega_{\star}$ and the value of maximum amplification is about twice that obtained from (19). 


\section{Discussion}

[23] The results obtained clearly demonstrate the essential difference between cyclotron amplification of a whistlermode wave propagating along the magnetic field for a smooth anisotropic distribution function for the energetic electrons (Figures 1 and 2) and for a distribution with a sharp velocity gradient ("step", Figures 3 and 4). In the case of the smooth distribution (4), we have moderate values of the one-hop amplification over a wide frequency band $\left(\Gamma_{\text {smooth }}<6\right.$, for reasonable values of the anisotropy parameter $\alpha<4)$. For the step-like distribution (16), a very high value of the one-hop amplification takes place over a very narrow frequency band.

[24] This shows the great advantage of a step-like distribution function over a smooth anisotropic function when seeking an explanation for discrete VLF emissions, for which a large value of the one-hop whistler-mode wave amplification is usually required to account for the observed emission properties. For example, the one-hop amplification by a step-like distribution at $L=3.4, W_{0}=18 \mathrm{keV}$, and $v_{\star} / v_{0}$ $\sim 0.2$ (these values were measured in the experiment of Bell et al. [2000], the value of $v_{\star}$ is estimated from the condition (18) for a top frequency of a hiss band) is an order of magnitude greater than the amplification by a smooth distribution function even at strong anisotropy $(\alpha=16)$. This theory can therefore explain the amplification which is needed for a satisfactory interpretation of the triggered VLF emissions observed by Bell et al. [2000], while to explain that with use of a smooth distribution demands extremely high values of the anisotropy index.

[25] Acknowledgments. This work was supported by NATO Linkage grant ESR.CLG 975144 and INTAS grant No. 99-00502. P.D.L., A.G.D. and V.Y.T. have been partly supported by the Russian Foundation for Basic Research, grant 99-02-16175a.

[26] Janet G. Luhmann thanks Manfred P. Leubner and another referee for their assistance in evaluating this paper.

\section{References}

Bell, T. F., U. S. Inan, and R. A. Helliwell, Simultaneous triggered VLF emissions and energetic electron distributions observed on Polar with PWI and HYDRA, Geophys. Res. Lett., 27(2), 165-168, 2000.

Bespalov, P. A., and V. Y. Trakhtengerts, The cyclotron instability in the
Earth radiation belts, in Reviews of Plasma Physics, vol. 10, edited by M. A. Leontovich, pp. 155-192, Plenum, New York, 1986.

Carlson, C. R., R. A. Helliwell, and U. S. Inan, Space-time evolution of whistler mode wave growth in the magnetosphere, J. Geophys. Res., 95(A9), 15,073-15,089, 1990.

Cornilleau-Wehrlin, N., J. Solomon, A. Korth, and G. Kremser, Experimental study of the relationship between energetic electrons and ELF waves observed on board GEOS: A support to quasilinear theory, $J$. Geophys. Res., 90(A6), 4141-4154, 1985.

Lyons, L. R., R. M. Thorne, and C. F. Kennel, Pitch angle diffusion of radiation belt electrons within the plasmapause, J. Geophys. Res., 77, 3455-3474, 1972.

Hobara, Y., V. Y. Trakhtengerts, A. G. Demekhov, and M. Hayakawa, Cyclotron amplification of whistler waves by electron beams in an inhomogeneous magnetic field, J. Geophys. Res., 103(A9), 20,449$20,458,1998$

Nunn, D., and S. S. Sazhin, On the generation mechanism of hiss-triggered chorus, Ann. Geophys., 9, 603-613, 1991.

Nunn, D., and A. J. Smith, Numerical simulation of whistler-triggered VLF emissions observed in Antarctica, J. Geophys. Res., 101(A3), 5261$5277,1996$.

Omura, Y., D. Nunn, H. Matsumoto, and M. J. Rycroft, A review of observational, theoretical and numerical studies of VLF triggered emissions, J. Atmos. Terr. Phys., 53, 351, 1991.

Smith, A. J., and D. Nunn, Numerical simulation of VLF risers, fallers and hooks observed in Antarctica, J. Geophys. Res., 103(A4), 6771-6784, 1998.

Tagirov, V. R., V. Y. Trakhtengerts, and S. A. Chernous, On the nature of pulsating auroral patches, Geomagn. Aeron., 26(4), 600-605, 1986.

Trakhtengerts, V. Y., and M. J. Rycroft, Whistler-electron interactions in the magnetosphere: New results and novel approaches, Geomagn. Aeron., 62(17-18), 1719-1733, 2000.

Trakhtengerts, V. Y., M. J. Rycroft, and A. G. Demekhov, Interrelation of noise-like and discrete ELF/VLF emissions generated by cyclotron interactions, J. Geophys. Res., 101(A6), 13,293-13,303, 1996.

Trakhtengerts, V. Y., A. G. Demekhov, D. L. Pasmanik, E. E. Titova, B. V. Kozelov, D. Nunn, and M. J. Rycroft, Highly anisotropic distributions of energetic electrons and triggered VLF emissions, Geophys. Res. Lett., 28(13), 2577-2579, 2001.

Villalón, E., and W. J. Burke, Theory of quasi-monochromatic whistler wave generation in the inner plasma sheet, J. Geophys. Res., 102(A7), $14,381-14,395,1997$.

A. G. Demekhov, D. L. Pasmanik, and V. Y. Trakhtengerts, Institute of Applied Physics, RAS, 46 Ulyanov Street, 603600 Nizhny Novgorod, Russia. (andrei@appl.sci-nnov.ru; pdl@aurora.appl.sci-nnov.ru; vyt@ appl.sci-nnov.ru)

D. Nunn, Department of Electronics and Computer Science, Southampton University, Southampton SO17 1BJ, UK. (dn@ecs.soton.ac.uk)

M. J. Rycroft, Faculty of Computer Sciences and Engineering, De Montfort University, Leicester LE 1 9BH, UK. (Michael.J.Rycroft@ ukgateway.net) 